\title{
Modulation of benzofuran structure as a fluorescent probe to optimize linear and nonlinear optical properties and biological activities
}

\author{
Przemysław Krawczyk ${ }^{1}$ (D)
}

Received: 30 June 2020 / Accepted: 13 September 2020 / Published online: 19 September 2020

(C) The Author(s) 2020

\begin{abstract}
The study presents the influence of structure modulation by introducing selected donor and acceptor substituents on optical properties of benzofuran used in biological imaging. As the starting form, 2-(5-formylbenzofuran-2-yl)acetamide described experimentally was used. This molecule contains an aldehyde group as reactive site, through which conjugation with protein occurs. Structure modulation was carried out by attaching additional electron-donating and electron-withdrawing substituents to the amino group, namely $-\mathrm{NH}_{2},-\mathrm{NHCH}_{3},-\mathrm{NO}_{2},-\mathrm{OH}$, and $-\mathrm{OCH}_{3}$. Studies have shown that the $-\mathrm{NH}_{2},-\mathrm{NHCH}_{3},-\mathrm{OH}$, and $-\mathrm{OCH} 3$ substituents do not induce a significant change in the position of maximum absorption and fluorescence relative to each other. They also do not change the parameters describing the nonlinear response. Only the presence of the $-\mathrm{NO}_{2}$ substituent results in significant solvatochromic shifts. Changing substituents also does not significantly affect the $\mathrm{LD}_{50}$ value, and all tested fluorescent probes should not be considered toxic to humans. Modulation of the benzofuran derivative structure also does not change the active center in which the biocomplex with the protein is formed. In each case, the conjugation takes place via LYS114. In addition, the study was prompted to analyze the linear and nonlinear optical properties of conjugates formed after the reaction with Concanavalin A.
\end{abstract}

Keywords Linear and nonlinear optical properties $\cdot$ Bioimaging $\cdot$ Conjugation with proteins $\cdot$ Biological activities $\cdot$ Toxicology $\cdot$ Solvatochromism · Benzofuran

$\begin{array}{ll}\text { Abbreviations } \\ \text { XCon } & \text { Marker determination after conjugation } \\ \text { ConA } & \text { Concanavalin A } \\ \text { THF } & \text { Tetrahydrofuran } \\ \text { MeAc } & \text { Acetone } \\ \text { MeCN } & \text { Acetonitrile } \\ \text { DMF } & \text { n,n-Dimethylformamide } \\ \text { DMSO } & \text { Dimethylsulfoxide }\end{array}$

Electronic supplementary material The online version of this article (https://doi.org/10.1007/s00894-020-04539-6) contains supplementary material, which is available to authorized users.

Przemysław Krawczyk przemekk@cm.umk.pl

1 Collegium Medicum, Faculty of Pharmacy, Department of Physical Chemistry, Nicolaus Copernicus University, Kurpińskiego 5 , 85-950 Bydgoszcz, Poland

\section{Introduction}

Benzofuran, because of its structure made of fused benzene and furan rings, can be classified as a heterocyclic organic compound. It is mainly utilized in the production of coumarone resins and it occurs naturally in the form of a colorless, oily liquid. Light oils obtained during coal tar distillation are the main source of this compound. There are several fields of application of benzofuran including organic synthesis, where it is used as a constituent for more complex systems, as well as material science. It is also widely found in many molecules that are biologically significant. Several different biological activities can be attributed to the derivatives of benzofuran. These include antiinflammatory, antioxidant, antitubercular, antiplasmodial, antitumor, antimicrobial, cytotoxic, enzyme inhibitory, HIV, and hepatitis $\mathrm{C}$ virus inhibitory activities [1-4]. The properties of benzofuran that are utilized in material science include on the other hand beneficial electrochemical behavior, thermal stability, high quantum yields, and blue-light emitting [5-7]. Also, their hole- 
transporting material properties $[8,9]$ make then useful for application as organic light-emitting diodes (OLED). Another interesting derivative is the benzofurannaphthyridine, which can be characterized with high fluorescence and quantum yield with solvatochromic properties [10]. Fluorobenzofuran on the other hand is used as a high triplet energy host material in the design of green phosphorescent OLEDs [11]. Recently, there are ongoing efforts to utilize benzofuran hydrazones as potential scaffolds in the development of multifunctional drugs [12]. The use of molecular docking studies also suggested antimicrobial and antifungal activity of the probe molecules $[13,14]$. Studies have also been carried out on the benzofuran derivative 2MBA in terms of its spectroscopic, quantum chemical, molecular docking, and drug likeness parameter properties. These studies suggested the pharmaceutical potential of the probe molecule $[15,16]$, while the molecular modeling studies on benzofuran derivatives indicate their potential as anticancer agents [17].

In the case of potential use of benzofuran derivatives as fluorescent probes, it is very important to study solvatochromic behavior. Such analysis should include an assessment of the polarity of the excited state, as they furnish information about the changes in electronic distribution and symmetry of the molecule in the excited state. The knowledge of the excited state dipole moment of the molecule would be helpful in explaining the nature of excited states, in describing the course of its photophysical/photochemical transformations, and in designing the nonlinear optical devices, and it allows one to judge the site of attack by electrophilic and nucleophilic reagents in photochemical reactions, etc. [18-28].

Currently, there is very little information in the literature on the subject of benzofuran derivatives in the context of their linear and nonlinear (NLO) optical properties. Moreover, there is no data on conjugates of these dyes with proteins and the impact of structure modulation on spectral and NLO properties. Due to the frequent use of benzofuran derivatives in the pharmaceutical and medical industry, the paper presents a detailed analysis of the 2-(5-formylbenzofuran-2yl)acetamide derivative. The analysis covers not only the molecule itself but also its derivatives resulting from the attachment of various electronodonor/acceptor substituents (Fig. 1). In addition, a thorough assessment of changes in the properties of the presented fluorescent probes after conjugation with the protein Concanavalin A (ConA) was performed. The choice of this derivative was not accidental. It is dictated by research carried out by Maridevarmath et al. [29], where a similar structure of 2-(5-methylbenzofuran-2-yl)acetohydrazide was analyzed. The differences consist in replacing the methyl group with an aldehyde group, through which the coupling with the protein occurs.

\section{Computational details}

All geometrical parameters of investigated molecules in their ground $\left(\mathrm{S}_{\mathrm{GS}}\right)$ and excited states $\left(\mathrm{S}_{\mathrm{CT}}\right)$ were calculated using the density functional theory approach with the PBE0 functional implemented in the Gussian09 program package [30] with the TIGHT optimization threshold option and the 6$311++\mathrm{G}(\mathrm{d}, \mathrm{p})$ basis set. In order to verify that all the structures correspond to the minima on the potential energy surface, an analysis of Hessians was performed. The electronic properties were characterized by computations of the vertical absorption and emission spectra, which were obtained using the timedependent density functional theory (TDDFT/PBE0) [31] and by including the state-specific (SS) corrected linear response (cLR) approach [32]. Due to the high compatibility of theoretical and experimental data [33-36], all spectroscopic calculations were performed using the PBE0 functional.

For the best consideration of the solvent impact on the fluorescence spectra, the ground state should be calculated with nonequilibrium solvation $[37,38]$. This was taken into account by including the state-specific (SS) corrected linear response (cLR) approach [32] to the theoretical calculations. In the SS approach, the solvent dynamic polarizations are determined by the difference of the electron densities of the initial and final states [39-41].

The dipole moments and polarities of the charge-transfer state $\left(\mathrm{S}_{\mathrm{CT}}\right)$ were evaluated by numerical differentiation of the excitation energies $(E)$ in the presence of an electric field $F$ of 0.001 a.u. strength:

$\Delta \mu_{g-C T}^{i}=\frac{E_{C T}\left(+F^{i}\right)-E_{C T}\left(-F^{i}\right)}{-2 F^{i}}-\frac{E_{g}\left(+F^{i}\right)-E_{g}\left(-F^{i}\right)}{-2 F^{i}}$

where $i$ stands for the Cartesian component of the dipole moment difference and $g$ is the ground state $\left(\mathrm{S}_{\mathrm{GS}}\right)$. The isotropic average polarizability $(\langle\alpha\rangle)$, polarizability anisotropy $(\Delta \alpha)$, and first-order hyperpolarizability $\left(\beta_{\mathrm{vec}}\right)$ were determined based on the Gaussian 09 program and defined as:

$\langle\alpha\rangle=\frac{\alpha_{x x}+\alpha_{y y}+\alpha_{z z}}{3}$
$\Delta \alpha=\sqrt{\frac{\left(\alpha_{x x}-\alpha_{y y}\right)^{2}+\left(\alpha_{x x}-\alpha_{z z}\right)^{2}+\left(\alpha_{y y}-\alpha_{z z}\right)^{2}+6\left(\alpha_{x y}^{2}+\alpha_{x z}^{2}+\alpha_{y z}^{2}\right)}{2}}$

$\beta_{\text {vec }}=\sum_{i=x, y, z} \frac{\mu_{i} \beta_{i}}{|\mu|}$

where $\beta_{i}(i=x, y, z)$ is given by $\beta_{i}=\left(\frac{1}{3}\right) \sum_{j=x, y, z}\left(\beta_{i j}+\beta_{j i j}+\beta_{j i j}\right)$

The density differences were obtained at the PBE0/6$311++\mathrm{G}(\mathrm{d}, \mathrm{p})$ level and are represented with a contour threshold of 0.02 a.u. In these graphs, the blue (purple) zones indicate density decrease (increase) upon electronic transition. The charge-transfer parameters, namely the charge-transfer 
Fig. 1 Structures of tested derivatives

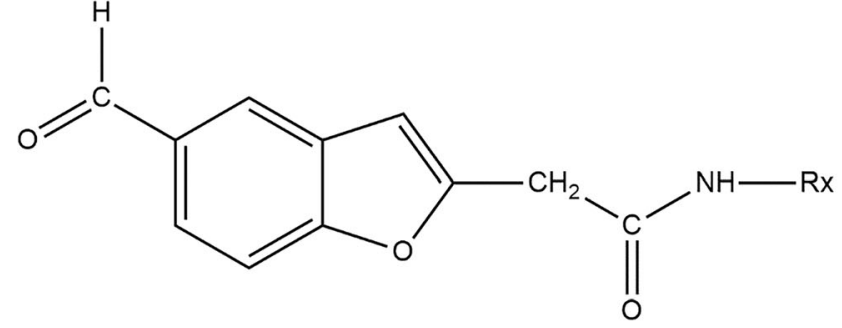

$\left(\mathrm{NH}_{2}\right)$

distance $\left(D_{\mathrm{CT}}\right)$ and the amount of transferred charge $\left(q_{\mathrm{CT}}\right)$, have been determined following a Le Bahers' procedure [42]. The solvent effect on the linear and nonlinear optical properties has been taken into account using the Integral Equation Formalism for the Polarizable Continuum Model (IEF-PCM) [43, 44].

Experimentally, the two-photon absorption (TPA) can be obtained by the dissipation of the incident light, which for a single-beam 2PA experiment is twice the transition rate. In this case, the two-photon cross section of the degenerate process is written as [45-47]:

$\sigma_{O F}^{(2)}=\frac{8 \pi^{3} \alpha^{2} \hbar^{3}}{e^{4}} \cdot \frac{\omega^{2} g(\omega)}{\Gamma_{F} / 2}\left\langle\delta^{O F}\right\rangle$

where $\alpha$ is a fine structure constant, $\omega$ is the frequency of absorbed photons (assuming one source of photons), $\Gamma_{\mathrm{F}}$ is the broadening of the final state (F) due to its finite lifetime, and $g(\omega)$ provides the spectral line profile, which often is assumed to be a $\delta$-function and $\left\langle\delta^{\mathrm{OF}}\right\rangle$ is the two-photon transition probability for the transition from the ground state to a final state.

In the case of a molecule absorbing two photons of the same energy in isotropic media, the degenerate $\left\langle\delta^{\mathrm{OF}}\right\rangle$ in an isotropic medium using a linearly polarized laser beam given by [48]:

$\left\langle\delta^{O F}\right\rangle=\frac{1}{15} \sum_{i j}\left[S_{i i}^{O F}\left(S_{j j}^{O F}\right)^{*}+2 S_{i j}^{O F}\left(S_{i j}^{O F}\right)^{*}\right]$

In this equation, $\mathrm{S}_{\mathrm{ij}}^{\mathrm{OF}}$ is the second-order transition moment given by:

$S_{i j}^{O F}\left(\zeta_{1}, \zeta_{2}\right)=\frac{1}{\hbar} \sum_{K}\left[\frac{\left\langle 0\left|\zeta_{1} \cdot \mu_{i}\right| K\right\rangle\left\langle K\left|\zeta_{2} \cdot \mu_{j}\right| F\right\rangle}{\omega_{\alpha}-\omega_{1}}+\frac{\left\langle 0\left|\zeta_{2} \cdot \mu_{i}\right| K\right\rangle\left\langle K\left|\zeta_{1} \cdot \hat{\mu}_{j}\right| F\right\rangle}{\omega_{\alpha}-\omega_{2}}\right]$

where $\hbar \omega_{1}+\hbar \omega_{2}$ should satisfy the resonance condition and $\left\langle 0\left|\zeta_{1} \cdot \widehat{\mu}_{i}\right| K\right\rangle$ stands for the transition moment between electronic states $\mid 0>$ and $|K\rangle$, respectively. $\zeta$ is the vector defining polarization of photons. To describe the two-photon allowed states, the quadratic response functions formalism [49, 50] within the DFT framework was used as implemented in the DALTON 2011 program [51, 52]. Solvent effects were taken into account with the self-consistent reaction field (SCRF) model. All the 2PA calculations were carried out employing the CAM-B3LYP functional and the 6-311++G(d,p) basis set.

The biological activities were simulated using a combination of the 3D/4D QSAR BiS/MC and CoCon algorithms [53-55].

The binding properties of considered dyes were studied by performing series of AutoDock 4.2 [56-58] and AutoDock Vina simulations. For each active complex comprising Concanavalin A (ConA), their crystal structures were taken from PDB ID: 2a7a [59]. The cubic grid box with the dimensions of $16 \AA$ and a grid spacing of $1 \AA$ was set up in such way that the reactive - $\mathrm{NH} 2$ groups of Lysine were at its center. In order to identify appropriate binding energy and conformation of compounds, the Lamarkian genetic algorithm was employed. For each atom of the receptor molecule, Gasteiger charges were calculated. The investigation of the binding site was performed using a united-atom scoring function. For each amino acid, the docking simulations were performed tenfold.

\section{Results}

\section{Physicochemical properties}

For all tested derivatives, the charge-transfer (CT) excitation corresponds to the HOMO $\rightarrow$ LUMO transition. In the case of $\mathrm{NH}_{2}$ molecule, energy separation between HOMOLUMO orbital ( $\mathrm{E}_{\mathrm{GAP}}$, Table S1) slightly decreases with increasing solvent polarity. However, for the $\mathrm{NH}_{2}$ derivative, $\Delta E_{G A P}^{T H F-\text { Water }}=0.03 \mathrm{eV}$. Changing the substituent also has little effect on these values. Only conversion of the $\mathrm{NH}_{2}$ substituent to $-\mathrm{NO}_{2}$ reduces $\mathrm{E}_{\mathrm{GAP}}$ and for water $\Delta E_{G A P}^{\mathrm{NH}_{2}-\mathrm{NO}_{2}}=0.0613 \mathrm{eV}$. After conjugation with the protein, the $\mathrm{E}_{\mathrm{GAP}}$ value increases on average by $0.6 \mathrm{eV}$. Only for the $-\mathrm{NO}_{2}$ substituent it is reduced by $0.3 \mathrm{eV}$. In addition, for most of the conjugates, $\mathrm{E}_{\mathrm{GAP}}$ also decreases with an increase of the medium polarity; for $\mathrm{NO}_{2} \mathrm{Con}$ this value increases. In addition, after the chemical hardness $(\eta)$ analysis, the tested derivatives should be treated as soft molecules. A high electronegativity $(\chi)$ value suggests an easy 
formation of covalent bonds during various chemical processes.

For the assessment of the sites of potential electrophilic and nucleophilic attack, the molecular electrostatic potential (MEP) analysis was performed, as seen in Fig. 2. For the $\mathrm{NH}_{2}$ derivative, it is the nitrogen atom with hydrogen attached to electron-donating group that is characterized with the highest electronegativity and it is therefore the most potent for a nucleophilic attack (positive, blue zones). Different substituents do not alter the location of these sites. On the other hand, the sites for electrophilic attack (negative, red, and yellow zones) are located mainly on the aldehyde group and the oxygen atom connected by a double bond with the carbon atom with attached $\mathrm{NH}_{2}$ group. As it was in the former case, changing the substituent does not result in changing of the location of these sites. When taking into account all the studied molecules, it is the $\mathrm{OH}$ derivative that can undergo an electrophilic attack most readily as the charge on the oxygen atom is as high as -0.09667 a.u. After conjugation with ConA, places for a potential nucleophilic attack do not change. However, the only site for an electrophilic attack is an oxygen atom near - $\mathrm{NH} 2$, while the zone at the markerprotein link disappears.

The studies have been devoted to many reflections on spectral properties, corresponding to the HOMO $\rightarrow$ LUMO photoexcitation $\left(\pi-\pi^{*}\right.$ transitions). In order to estimate contributions from other orbitals and determine the nature of electronic states, the density variation upon photoexcitation $(\Delta \rho(\mathrm{r}))$ was computed for the first electronic transitions, which is graphically depicted in Fig. 3. For BIN-T, the figure indicates that the depletion zones (blue) are mainly located on aldehyde group, while in contrast, growth zones (purple) on benzofuran part. The presence and change of donor-acceptor substituents does not change the position of these zones. The exception is the $\mathrm{NO}_{2}$ derivative, for which depletion zones include not only the aldehyde group but also benzofuran part. In contrast,
Fig. 2 The MEP surfaces for benzofuran derivatives in water. Values are given in [a.u]

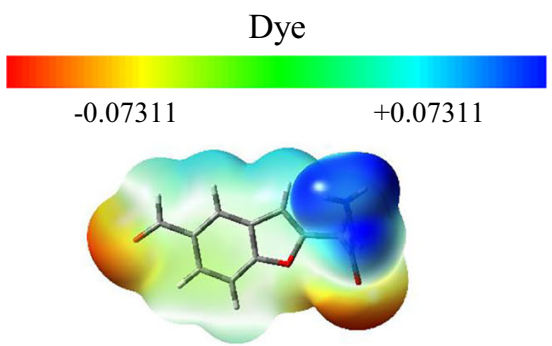

$+0.07133$

NHR

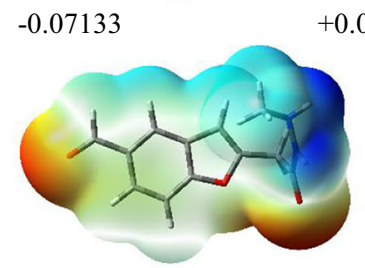

$-0.07653$

$+0.07653$

$\mathrm{NO}_{2}$

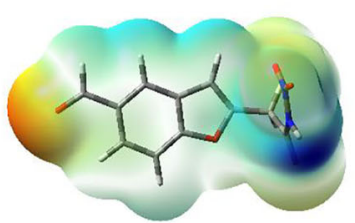

$-0.09667$

$+0.09667$

$\mathrm{OH}$

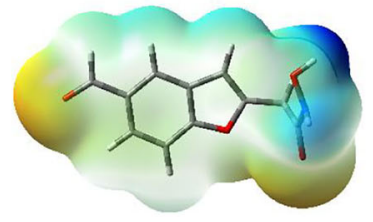

$-0.06656$

$+0.06656$

OR

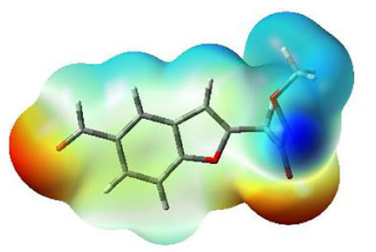

Conjugate

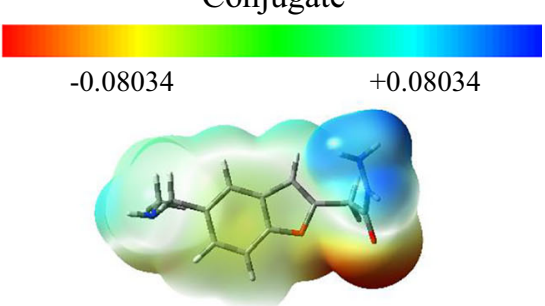

$+0.08054$
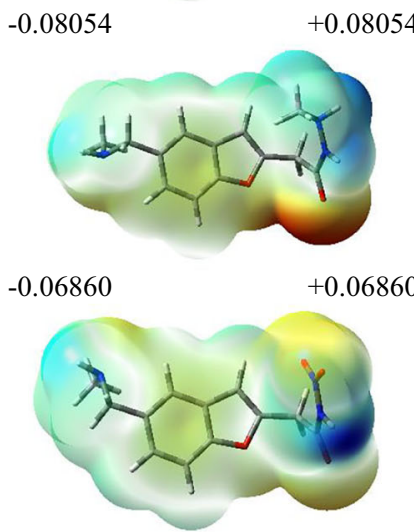

$-0.08952$

$+0.08952$

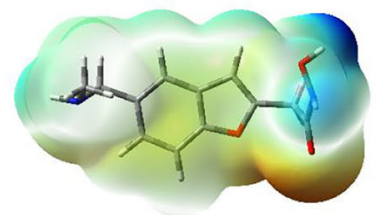

$-0.07597$ $+0.07597$

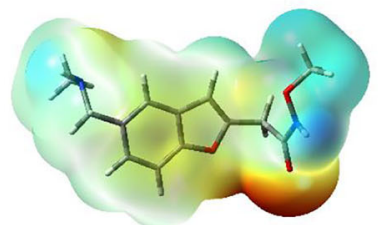


Fig. 3 Density difference plot in water. The purple and green lobes correspond to $\left(\rho_{-}\right)$and $\left(\rho_{+}\right)$, respectively

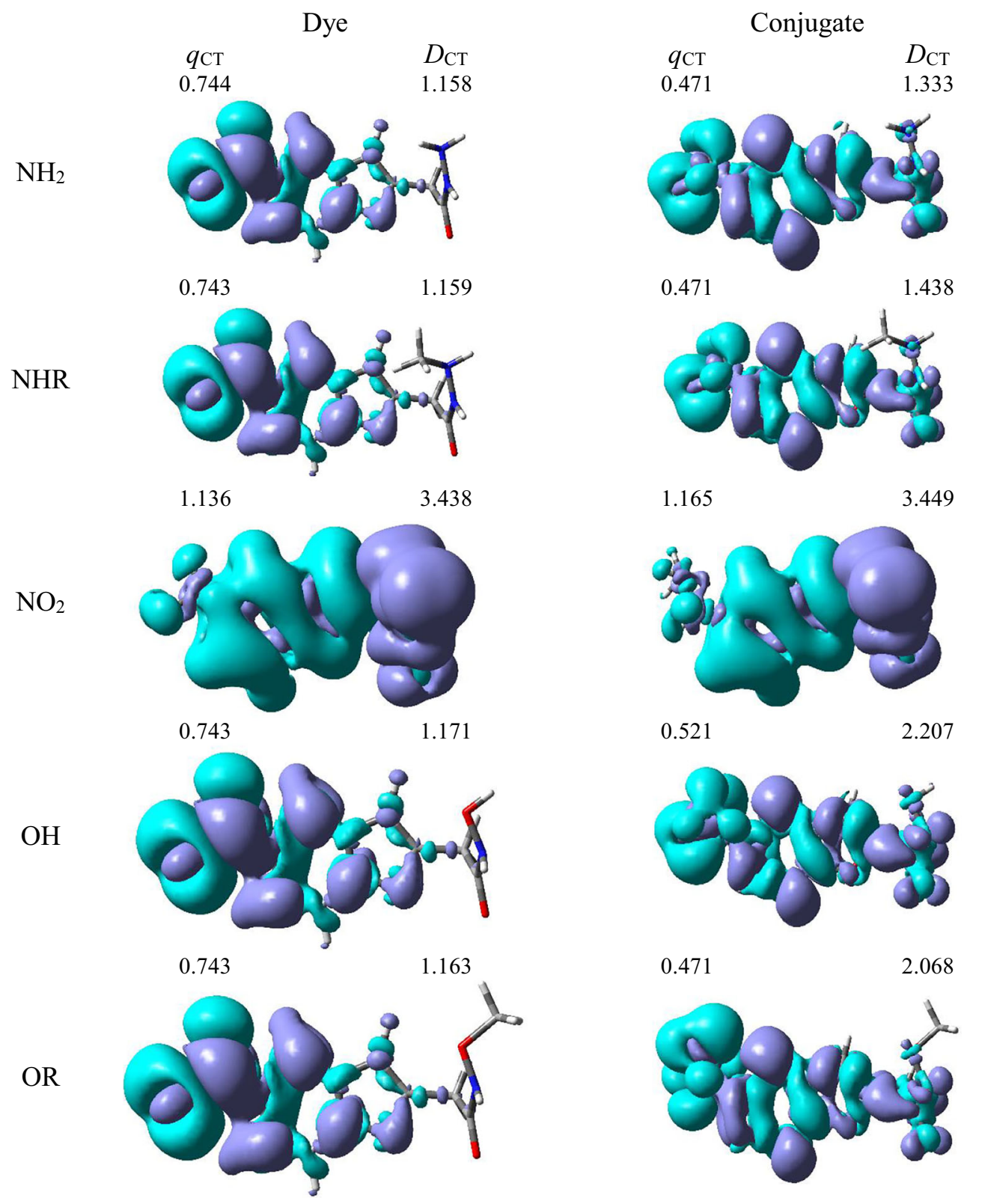

purple zones extend mainly to parts with an electronwithdrawing substituent. Protein conjugation does not significantly change the position of these zones.

On the other hand, the polarity of the environment does not significantly affect the parameters describing $\Delta \rho(\mathrm{r})$ (Table S3). For all derivatives, the amount of the transferred charge is not variable as a function of solvent polarity and is at 0.743-0.744 a.u. level. As for previous quantities, the exception is the NO2 marker, for which $q_{\mathrm{CT}}$ is 1.136 a.u. The polarity of the environment also significantly affects the chargetransfer distance and increases it from $1.118 \AA$ in THF to $1.158 \AA$ in water for $\mathrm{NH}_{2}$ molecule. For other derivatives, the $D_{\mathrm{CT}}$ value is at a similar level and for example a difference $\Delta D_{C T}^{O R-N H_{2}}$ is only $0.005 \AA$. However, for the $\mathrm{NO}_{2}$ derivative, the $D_{\mathrm{CT}}$ value is three times higher than the other molecules and exceeds $3.4 \AA$. Conjugation reduces the $q_{\mathrm{CT}}$ value by almost half and for $\mathrm{NH}_{2}$ in water $\Delta q_{C T}^{\mathrm{NH}_{2}-\mathrm{NH}_{2} \mathrm{Con}}$ is 0.273 a.u. (Tab. S4). The exception is again $\mathrm{NO}_{2}$, for which these values remain practically unchanged. In addition, $D_{\mathrm{CT}}$ increases after conjugation with the protein. While for $\mathrm{NH}_{2}$

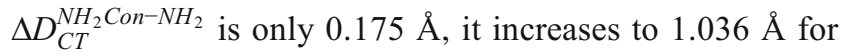
the $\mathrm{OH}$ derivative. Solvent effects on $\Delta \rho(\mathrm{r})$ parameters remain the same as for markers before combining with the macromolecule. The presented analysis indicates the charge-transfer nature of tested derivatives. It also confirms the contributions from the HOMO-LUMO transition. At the same time, it indicates the possibility of the presence of contributions from other orbitals. 
All tested markers are characterized by good solubility in all discussed media (Tab. S). $\Delta G_{\text {solv }}$ increases with the increase in the polarity of the environment. Only the transition from DMSO to water creates a slight decrease and for $\mathrm{NH}_{2}$ $\Delta \Delta G_{\text {sol }}$ is $1.51 \mathrm{kcal} / \mathrm{mol}$. At the same time, $\Delta G_{\text {sol }}$ in water is slightly lower than the values in THF and $\Delta \Delta G_{\text {sol }}$ is $0.55 \mathrm{kcal} / \mathrm{mol}$. After conjugation, the solubility practically does not change, and the $\Delta G_{s o l}$ value increases slightly (Tab. S6). For the reference $\mathrm{NH}_{2}, \Delta \Delta G_{\text {sol }}$ in THF is $0.07 \mathrm{kcal} / \mathrm{mol}$ and increases to $0.47 \mathrm{kcal} / \mathrm{mol}$ in water. Identical relationships are observed for the remaining markers.

\section{Linear optical properties}

Theoretical linear optical properties of analyzed markers are shown in Tables 1 and SI7. Due to the high compatibility of theoretical and experimental values obtained on the basis of PBE0 [33-36], the spectroscopic parameters were determined using this function. It also accurately predicts $\mathrm{NH}_{2}$ values recorded experimentally. For vertical values, the $\Delta \lambda_{A B S}^{T D D F T-E X P}$ difference in THF is $23.46 \mathrm{~nm}$ but in DMF it is only $1.10 \mathrm{~nm}$. For the values obtained within the cLR approximation, this error increases to the levels of $25.46 \mathrm{~nm}$ and $5.83 \mathrm{~nm}$, respectively. In the case of benzofuran derivatives, the presence of donor-acceptor substituents results in week shift in absorption maximum $\left(\lambda_{\mathrm{ABS}}\right)$. Attaching a methyl group to the $-\mathrm{NH}_{2}$ results in a batochromic shift. However, the magnitude of this shift is insignificant and for vertical values is only $0.06 \mathrm{~nm}$ on average. In turn, the replacement of the amino substituent with $-\mathrm{OH}$ also shifts $\lambda_{\mathrm{ABS}}$ in the direction of longer wavelengths by $0.20 \mathrm{~nm}$ on average. Attaching an additional $-\mathrm{CH}_{3}$ group to the hydroxyl substituent results in a hypsochromic shift and $\Delta \lambda_{\mathrm{ABS}}$ is on average $0.07 \mathrm{~nm}$. For the investigated benzofuran derivatives, the largest solvatochromic shift is observed in $\mathrm{MeCN}$ and the smallest in DMS. Nevertheless, conversion of $-\mathrm{NH}_{2}$ with $\mathrm{NHCH}_{3},-\mathrm{OH}$, and $-\mathrm{OCH}_{3}$ substituents does not cause significant changes in the location of the maximum absorption in all analyzed solvents. A significant shift is obtained when substituting the amino group with a $-\mathrm{NO}_{2}$ group. The average value of the batochromic shift $\Delta \lambda_{A B S}^{\mathrm{NO}_{2}-\mathrm{NH}_{2}}$ is at the level of $30.30 \mathrm{~nm}$. Identical conclusions can be drawn after analyzing the maintained values within the cLR approximation. Conjugation with ConA is followed by another solvatochromic shift. In the case of $\mathrm{NH}_{2}, \mathrm{NHR}, \mathrm{OH}$, and $\mathrm{OR}$ derivatives, a shift towards shorter waves is observed. The size of this slide is significant and amounts to an average of $54 \mathrm{~nm}$. In turn, there is a batchromic shift for the $\mathrm{NO}_{2}$ probe, however its size is smaller compared with other probes and on average is $27 \mathrm{~nm}$.

The position of maximum absorption is slightly sensitive to environmental changes, for both initial markers and conjugates. The $\lambda_{\mathrm{ABS}}$ value monotonously decreases as the polarity of the medium increases. However, the transition from weakly polar THF to water is accompanied by an increase in excitation energy of just $1.5 \mathrm{~nm}$. Also, $\Delta \lambda_{A B S}^{\text {marker-conjugate }}$ decreases with increasing solvent polarity.

The interconnectedness of the analysis of spectroscopic parameters with MEP indicates the possibility of specific interactions in the solute-solvent system. A monotonic increase in excitation energy indicates on larger polarization and better $\mathrm{S}_{\mathrm{g}}$ stabilization. However, this is not consistent with the polarity of the excited state $\left(\Delta \mu_{C T-g}\right)$. Hypsochromic shift, as the effect of the increase in environmental polarity, should result in the $\mu_{\mathrm{g}}>\mu_{\mathrm{CT}}$ relationship. In contrast, for both markers and conjugates, there is an inverse relationship (Tables S11 and S12), which is a characteristic of positive solvatochromism. In any case, the polarity of the excited state also changes monotonously. In all media, the highest $\Delta \mu_{C T-g}$ value is a characteristic for $\mathrm{NO}_{2}$ marker and in terms of average values probes can be ranked as follows: $\mathrm{NO}_{2}\left(\Delta \mu_{C T-g}=\right.$ $10.98 \mathrm{D})>\mathrm{OH}=\mathrm{OR}\left(\Delta \mu_{C T-g}=8.30 \mathrm{D}\right)>\mathrm{NH}_{2}=\mathrm{NHR}$ $\left(\Delta \mu_{C T-g}=7.20 \mathrm{D}\right)$. In addition, as the polarity of the medium increases, the dipole moment values increase and both $\mu_{g}^{\text {Water-THF }}$ and $\mu_{C T}^{\text {Water-THF }}$ do not exceed $0.8 \mathrm{D}$. In terms of $\mu_{\mathrm{g}}$ value, the molecules can be ordered as follows: $\mathrm{OR}>\mathrm{NH}_{2}$ $=\mathrm{NHR}>\mathrm{OH}>\mathrm{NO}_{2}$, and for $\mu_{\mathrm{CT}}$ : $\mathrm{OR}>\mathrm{OH}>\mathrm{NHR}>\mathrm{NH}_{2}>$ $\mathrm{NO}_{2}$. The polarity of the excited state decreases after conjugation. The smallest differences in $\Delta \Delta \mu_{C T-g}$ are visible for the $\mathrm{NH}_{2}$ derivative $(1.27 \mathrm{D})$, and the largest for $\mathrm{OR}(2.61 \mathrm{D})$.

Table 1 Linear and nonlinear optical properties in water

\begin{tabular}{|c|c|c|c|c|c|c|c|c|c|c|c|}
\hline & $\lambda_{A B S}^{T D D F T}$ & $f$ & $\lambda_{A B S}^{c L R}$ & $\mu_{\mathrm{GS}}$ & $\mu_{\mathrm{CT}}$ & $\lambda_{F L}^{T D D F T}$ & $\langle\alpha\rangle$ & $\Delta \alpha$ & $\beta_{v e c}$ & $\left\langle\delta^{O F}\right\rangle$ & $\sigma_{O F}^{(2)}$ \\
\hline $\mathrm{NH}_{2}$ & 313.94 & 1.1132 & 317.10 & 9.50 & 16.31 & 396.32 & 212.90 & 96.33 & 669.93 & 0.06 & 0.00 \\
\hline NHR & 313.98 & 0.7062 & 317.12 & 9.43 & 16.89 & 396.33 & 228.99 & 92.95 & 610.60 & 0.06 & 0.00 \\
\hline $\mathrm{NO}_{2}$ & 314.12 & 1.0190 & 317.28 & 5.26 & 15.44 & 434.56 & 223.63 & 82.90 & 526.91 & 626.96 & 2.18 \\
\hline $\mathrm{OH}$ & 314.09 & 1.0666 & 317.23 & 9.28 & 17.69 & 396.67 & 207.11 & 102.87 & 713.63 & 0.05 & 0.00 \\
\hline OR & 344.42 & 1.0461 & 338.14 & 9.72 & 18.12 & 396.64 & 223.56 & 96.77 & 639.03 & 0.06 & 0.00 \\
\hline $\mathrm{NH}_{2} \mathrm{Con}$ & 259.34 & 1.2824 & 258.88 & 8.01 & 13.91 & 356.88 & 233.11 & 92.48 & 0.75 & 18.05 & 0.08 \\
\hline NHRCon & 259.44 & 1.2661 & 259.14 & 8.08 & 13.91 & 356.89 & 249.23 & 76.22 & 1.02 & 17.65 & 0.08 \\
\hline $\mathrm{NO}_{2} \mathrm{Con}$ & 260.39 & 1.2749 & 260.64 & 2.43 & 21.68 & 412.67 & 244.02 & 72.19 & 3.31 & 1204.90 & 3.82 \\
\hline OHCon & 259.90 & 1.1615 & 260.37 & 6.86 & 12.70 & 357.23 & 227.49 & 99.17 & 0.12 & 32.85 & 0.15 \\
\hline ORCon & 371.08 & 1.0435 & 365.39 & 5.58 & 11.02 & 357.20 & 243.36 & 90.25 & 1.88 & 32.85 & 0.15 \\
\hline
\end{tabular}


Only for the $\mathrm{NO}_{2}$ probe, an increase in CT state polarity is observed, by $8.98 \mathrm{D}$ on average. This is due to changes in dipole moment values. In all cases, $\mu_{\mathrm{g}}$ and $\mu_{\mathrm{CT}}$ decreases. Only for $\mathrm{NO}_{2}, \mu_{\mathrm{CT}}$ increases by an average of $6.27 \mathrm{D}$.

Tables 1 and S13 show the values of the de-excitation energy $\left(\lambda_{\mathrm{FL}}\right)$. Also, in this case the functional PBE0 perfectly reproduces the values and $\Delta \lambda_{F L}^{T D D F T-E x p}$ is only $3.35 \mathrm{~nm}$. As for $\lambda_{\mathrm{ABS}}$, the location of maximum fluorescence for $\mathrm{NH}_{2}$, $\mathrm{NHR}, \mathrm{OH}$, and OR derivatives lies in close proximity. The $\mathrm{OH}$ spectrum is the most shifted towards longer wavelengths, while $\mathrm{NH}_{2}$ is the most shifted towards shorter wavelengths. However, the $\Delta \lambda_{F L}^{O H-N H_{2}}$ difference is only $0.34 \mathrm{~nm}$. The most batochromic shifted spectrum is $\mathrm{NO}_{2}$ and $\Delta \lambda_{\mathrm{FL}}^{\mathrm{NO}_{2}-\mathrm{NH}_{2}}$ is $40.98 \mathrm{~nm}$. Also in this case, the energy of de-excitation increases monotonously as a function of the polarity of the medium. However, again these differences are insignificant and $\Delta \lambda_{F L}^{T H F-\text { Water }}$ for $\mathrm{NH}_{2}$ is $5.30 \mathrm{~nm}$. The largest solvatochromic shift is observed for the NO2 probe, where $\Delta \lambda_{F L}^{T H F-\text { Water }}=$ $12.13 \mathrm{~nm}$. Conjugation promotes an additional increase in de-excitation energy. The size of the hypsochromic shift is the smallest for $\mathrm{NO}_{2}$ and is on average $19.70 \mathrm{~nm}$. For other probes, it is at the same level and amounts to $39.30 \mathrm{~nm}$.

All tested derivatives have a high Stokes shift value $\left(\Delta \nu^{S t}\right)$, in the range $6500-6800 \mathrm{~cm}^{-1}$. In each case, its value decreases monotonously as a function of the solvent polarity. However, the transition from THF to water decreases the $\Delta \nu^{S t}$ value by only $180 \mathrm{~cm}^{-1}$. Only for $\mathrm{NO}_{2}$, this decrease is greater and $\Delta \Delta \nu^{S t}$ is $571.88 \mathrm{~cm}^{-1}$. In terms of this quantity, the analyzed probes can be ranked as follows: $\mathrm{NO}_{2}<\mathrm{NHR}<\mathrm{NH}_{2}<\mathrm{OH}<\mathrm{OR}$, with $\Delta \nu_{O R-N H R}^{S t}=11.01 \mathrm{~cm}^{-1}$. After conjugation with ConA, the $\Delta \nu^{S t}$ value for $\mathrm{NO}_{2}$ decreases by $3186.65 \mathrm{~cm}^{-1}$ on average. For the remaining probes, this quantity increases, and the largest increase is observed for $\mathrm{NH}_{2}\left(3951.81 \mathrm{~cm}^{-1}\right)$. Although all derivatives are described by a high $\Delta \nu^{S t}$ value, the presence of $\mathrm{NO}_{2}$ will be the least desirable substituent in the marker structure. However, taking into account all linear optical parameters, it allows obtaining a probe with a different spectrum of operation.

\section{Nonlinear optical properties}

The polarizability $(\langle\alpha\rangle)$ and first hyperpolarizability $\left(\beta_{\mathrm{vec}}\right)$ of molecules irradiated with intense laser light giving the electric field is the subject of many research in terms of understanding various nonlinear optical properties (NLO). In particular, these studies include the interrelationship of NLO with the electronic structure to design new multifunctional fluorescence probes. The calculated values of $\langle\alpha\rangle$ and $\beta_{\mathrm{vec}}$ are collected in Tables 1, S15, and S16. In the case of $\langle\alpha\rangle$, the analyzed compounds can be ordered in the following way: $\langle\alpha\rangle^{O H}$ $<\langle\alpha\rangle^{N H_{2}}<\langle\alpha\rangle^{O R}<\langle\alpha\rangle^{N O_{2}}<\langle\alpha\rangle^{N H R}$. With the increase in the solvent polarity, the polarizability value increases and the transition from THF to water is accompanied by an average increase of 12 a.u. At the same time, the polarity of the medium does not affect the alignment of the studied molecules in terms of $\langle\alpha\rangle$. After the reaction with ConA, the polarizability value increases on average by 19-21 a.u. Like the solvent power, conjugation does not affect the ranking of the tested derivatives. For the first hyperpolarizability, benzofuran derivatives can be ordered inversely to $\langle\alpha\rangle$ : $\beta_{v e c}^{N O_{2}}<\beta_{v e c}^{N H R}<\beta_{v e c}^{O R}<$ $\beta_{v e c}^{\mathrm{NH}_{2}}<\beta_{\text {vec }}^{\mathrm{OH}}$. Also in this case, the $\beta_{\text {vec }}$ value increases monotonously as the medium permeability increases, and the transition from THF to water increases in the range of 111 a.u. up to 127 a.u. More importantly, for each derivative after conjugation $\beta_{\text {vec }}$ drops below 4 a.u. Since the nonlinear response will be derived from the structure obtained after conjugation with protein, markers based on the benzofuran backbone are not suitable for NLO processes. At the same time, in this case the power of the donor/acceptor substituents does not affect the intensification of the nonlinear response, and thus it cannot be efficient e.g. in second-harmonic generation (SHG).

Table S1 shows the value of two-photon absorption cross section (TPA, $\left.\left\langle\delta^{O F}\right\rangle\right)$. In general, it is difficult to decisively conclude about the effect of the solvent on the $\left\langle\delta^{O F}\right\rangle$ value. For derivatives before the reaction with ConA, these values are practically zero and only for $\mathrm{NO}_{2}$ in water a slight increase is observed. However, $\left\langle\delta^{O F}\right\rangle$ is still very small and is only 626 a.u. Conjugation becomes a factor that maximizes the value of TPA. However, in all media, $\left\langle\delta^{O F}\right\rangle$ does not exceed the value of 33 a.u. Again, the largest increase is observed for the $\mathrm{NO}_{2}$ derivative, because for this molecule a larger increase is visible and $\mathrm{D}\left\langle\delta^{O F}\right\rangle$ reaches the value of 1204.90 in water. In this case, the TPA value also increases monotonously as a function of solvent polarity. However, in order to compare the calculated values of the TPA with those determined experimentally $\left(\sigma_{O F}^{(2)}\right)$, the relation 5 was used. In this equation, the broadening of the final state due to its finite lifetime $0.25 \mathrm{eV}$ was assumed. The effect of the solvent on the $\sigma_{O F}^{(2)}$ value remains the same as for $\left\langle\delta^{O F}\right\rangle$. For all molecules, except $\mathrm{NO}_{2}, \sigma_{O F}^{(2)}$ is $0 \mathrm{GM}$. For this compound, only in water the value is greater than $0 \mathrm{GM}$ and is $2.18 \mathrm{GM}$. Conjugation is not conducive to improving these values. Only for $\mathrm{NO}_{2} \mathrm{Con}$ the $\sigma_{O F}^{(2)}$ increase is observed; however, it is small and in THF is only 3.65 GM and in water 1.64 GM. Based on this analysis, to obtain a probe with a high TPA value, used as tools in real-time dynamic in vivo and in vitro research, an additional chromophore group intensifying the NLO response should be added to the structure of the analyzed dyes. In particular, it will be desirable to modify the derivative containing the $-\mathrm{NO}_{2}$ substituent.

\section{Biological activities}

The tested derivatives are described by relatively good bioavailabilty $(\log P>2.5)$. This suggests good permeability 
Fig. 4 Theoretically determined toxicological parameters. Blue line, markers before conjugation; green line, conjugates
$\mathrm{NH} 2$

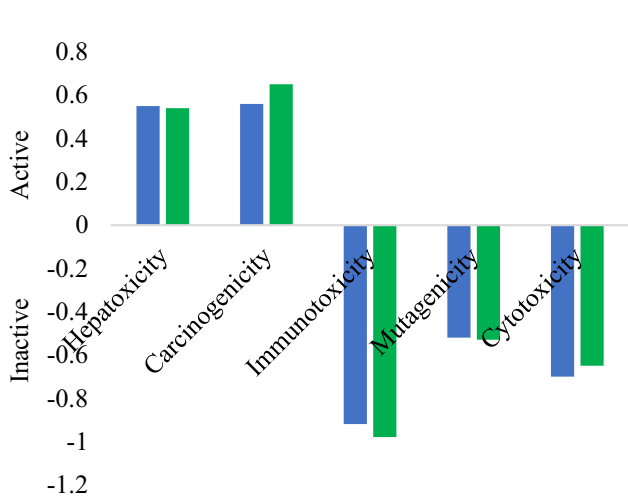

$\mathrm{NO} 2$

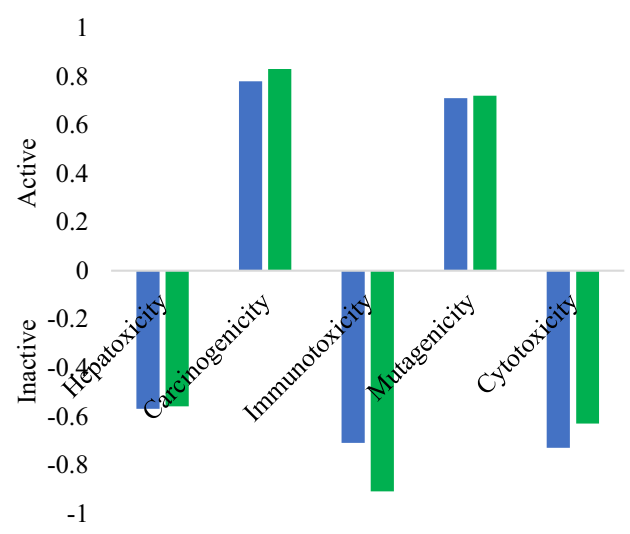

NHR

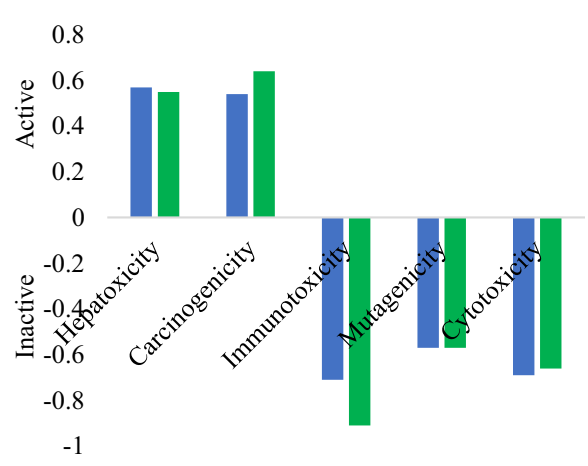

$\mathrm{OH}$

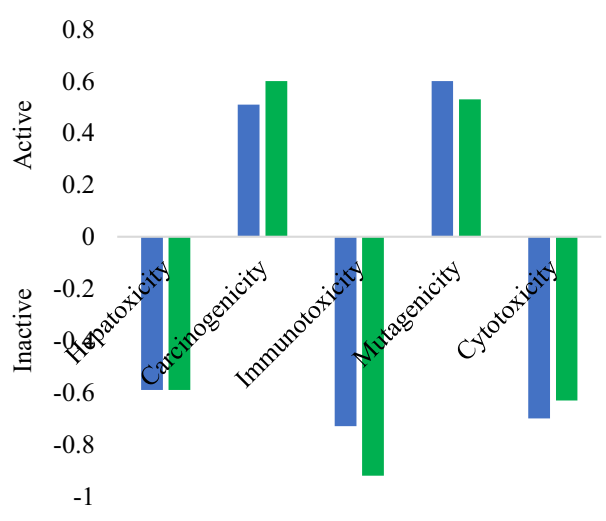

OR

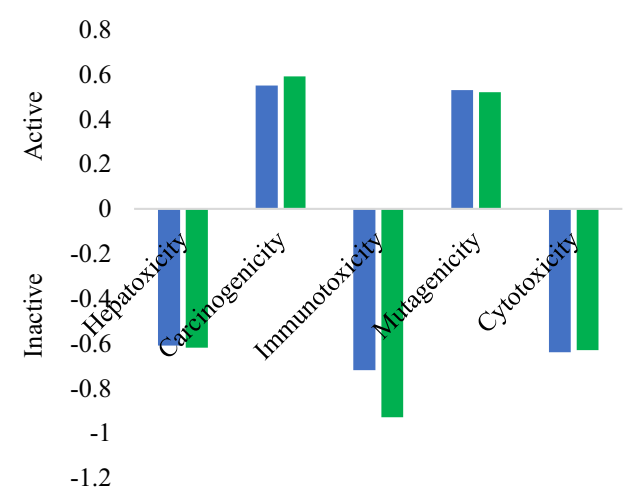

through cell membranes and achieving adequate concentration at the site of interaction with proteins. After conjugation, this value exceeds 3.5. Only for OHCon and ORCon it drops to 1.08 and 1.80 , respectively. A calculated $\operatorname{LogBCF}$ value in the range of 2.1-2.3 indicates the lack of bioaccumulation in the tissues of living organisms and the ease of excretion with urine. At the same time, the reaction with ConA does not affect the value of $\operatorname{LogBCF}$. For this reason, all derivatives should not bioaccumulate after fulfilling their optical role. Moreover, the tested molecules before and after conjugation are characterized by high metabolism by CYP450-2D6 and
Cyp450-3A4 (Tables S17 and S18). The above observations indicate the fact that both forms of markers will be promptly removed from the tissues and will not interact with different drugs or biomolecules. The oral toxicity value calculated as $\mathrm{LD}_{50}>1800 \mathrm{mg} / \mathrm{kg}$ classifies the studied derivatives in class 4 of the degree of toxicity which means that they can be regarded as essentially nontoxic for humans. The toxicity of the studied molecules is not influenced by the presence of different substituents; however, they do alter their other toxicological parameters (Fig. 4). None of the derivatives shows immunotoxicity and cytotoxicity. However, all derivatives 
Fig. 5 Results of the AutoDock simulations

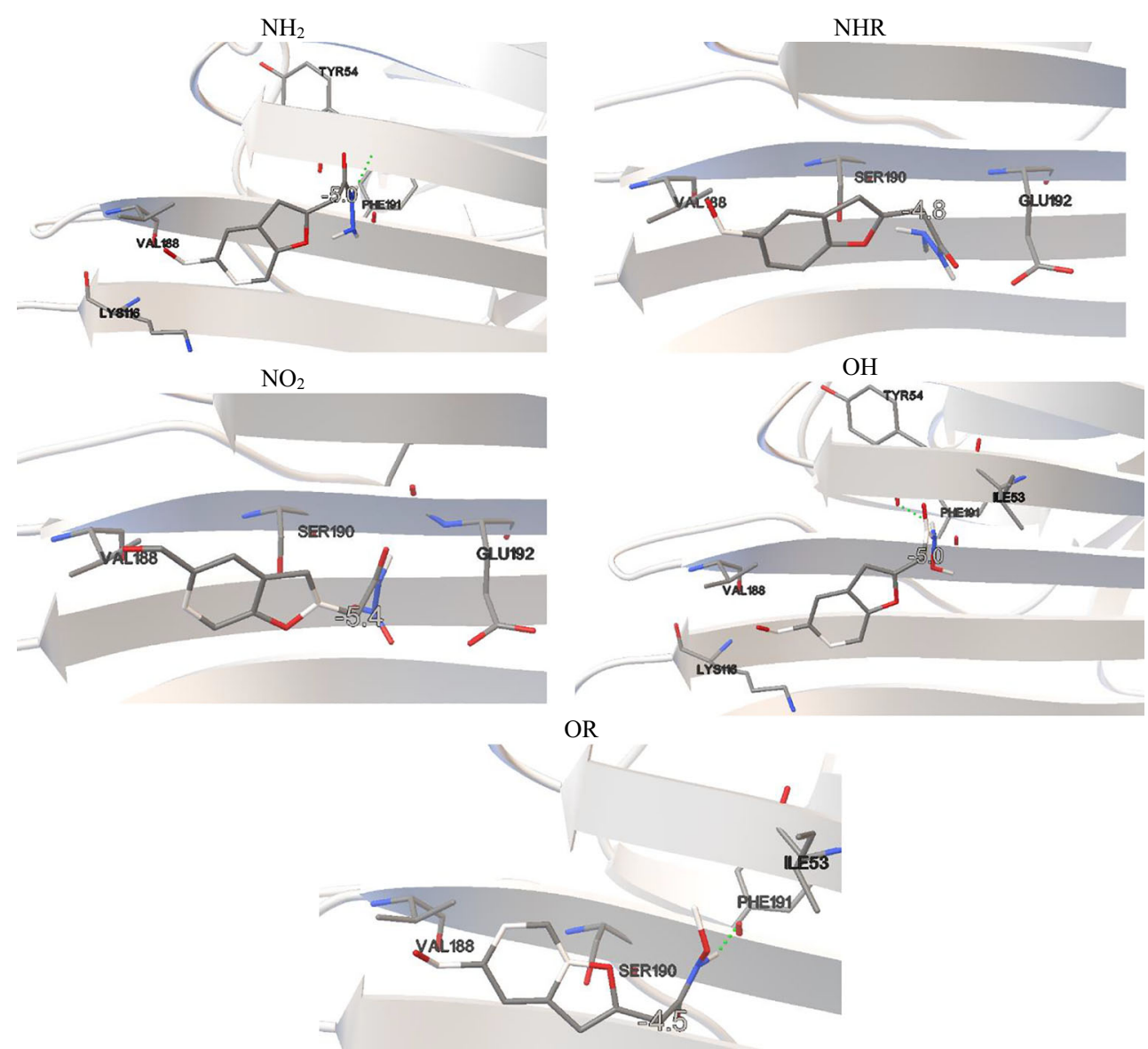

exhibit carcinogenicity properties. The presence of the $\mathrm{NH}_{2}$ and $-\mathrm{NHCH}_{3}$ substituents causes hepatoxicity but no mutagenicity. Other derivatives are characterized by inverse relationships. In addition, conjugation does not affect the reported toxicity properties. On the other hand, all molecules, before and after attachment to a macromolecule, have other biological activities suggesting their potential use in other areas of medicine. All derivatives, except $\mathrm{NO}_{2}$, are characterized by high antibacterial activity $(P>70 \%)$. However, only the $\mathrm{NO}_{2}$ derivative has antioxidant properties $(P>57 \%)$. Conjugation with ConA causes antioxidant properties to occur in all molecules, and there is no change in the antibacterial property. In addition, the tested benzofuran derivatives are characterized by many other activities, such as alpha-radioprotector activity, acyl-CoA-holesterol transferase inhibitory activity, gamma-radioprotector activity mechanism I, antiadenovirus activity, antipsychotic activity diazepine site, antitumor alkylic activity, antitumor DNA antimetabolitic activity, antitumor topoisomerase I inhibitory activity, and HIV1-proteaze inhibitory activity. At the same time, the influence of subsequent substituents on the maximization or reduction of these activities is demonstrated in Tables S17 and S18.

\section{AutoDock simulations}

As it was stated before, the considered benzofuran derivatives cannot be used in the studies on two-photon absorption. They can be utilized however as fluorescent markers in single-photon studies, which makes them suitable for application as alternative markers in fluorescent probes used for medical imaging. It is the reaction with lysine through which the conjugation with the protein occurs. For this particular study, Concanavalin A was chosen and the conjugation to ConA molecule occurs via LYS114 (Fig. 5). The presence of different substituents does not change the active binding site of the studied derivatives to the protein molecule. Among studied derivatives, the highest affinity for this active center is shown by the $\mathrm{NO} 2$ derivative. In this case, the binding energy $\left(\Delta G_{b}\right)$ was calculated to be $5.4 \mathrm{kcal} / \mathrm{mol}$ (Table S19), while the inhibition constant $\left(K_{i}\right)$ of this binding site was found to be $1.54 \mu \mathrm{M}$. An aromatic cage formed by LYS114, VAL188, SER190, and GLU192 is the site for the insertion of the molecule. No $\pi-\pi^{*}$ interactions were found the active site, neither it is stabilized by H-bonds. Other two derivatives, namely $\mathrm{NH}_{2}$ and $\mathrm{OH}$, are characterized by a slightly lower affinity for ConA with $\Delta G_{b}=-5.0 \mathrm{kcal} / \mathrm{mol}$ and the corresponding inhibition constants equal $2.19 \mu \mathrm{M}$ and $2.47 \mu \mathrm{M}$, respectively. 
The active impact cavity here is LYS116, VAL188, TYR54, and PHE191. In the case of OH, ILE53 also interacts here. In this case, hydrogen bonds are observed but no $\pi-\pi^{*}$ interactions. For the first molecule, the H-bond is formed between the hydrogen atom of the first $\mathrm{NH}$ group at the donor substituent and the nitrogen atom TYR54. In the second case, the same hydrogen will interact with the oxygen atom TYR54. OR has the lowest affinity for the protein, for which $\Delta G_{b}=-$ $4.5 \mathrm{kcal} / \mathrm{mol}$ and $K_{i}=5.14 \mu \mathrm{M}$. During conjugation, the $\mathrm{C}=\mathrm{O}$ bond raptures and then a new one forms with the - $\mathrm{NH} 2$ group of protein. The rate of biocomplex creation will therefore also be affected by the energy barrier $(\Delta E)$ necessary to overcome. The lowest $\Delta E$ value when moving the oxygen atom away from the aldehyde group at a distance of $2.3 \AA$ is observed for $\mathrm{NO}_{2}$ derivative and is $20.22 \mathrm{kcal} / \mathrm{mol}$. A slightly higher value is observed for $\mathrm{NH}_{2}$ and $\mathrm{NHr}$ for which $\Delta E$ is $22.13 \mathrm{kcal} / \mathrm{mol}$ and $22.14 \mathrm{kcal} / \mathrm{mol}$, respectively. The strongest bond, and thus hindering the conjugation, is the $\mathrm{C}=\mathrm{O}$ in $\mathrm{OH}$ and $\mathrm{OR}$, where $\Delta E$ is $23.16 \mathrm{kcal} / \mathrm{mol}$ and $23.26 \mathrm{kcal} / \mathrm{mol}$, respectively. In addition, no significant structural changes are observed for any molecule due to matching with the aromatic cavity.

\section{Conclusions}

This paper presents the effect of modulation of benzofuran derivatives structure to optimize linear and nonlinear optical properties and biological activities. The obtained results were compared with those obtained for probes after conjugation with Concanavalin A. Regardless of the type of substituent used, the CT excitation corresponds to the HOMO-LUMO transition. Analysis of optical properties showed that the $\mathrm{NHCH}_{3}$, - $\mathrm{OH}$, and - $\mathrm{OCH}_{3}$ substituents do not induce significant batochromic shifts of maximum absorption and fluorescence relative to the reference $-\mathrm{NH}_{2}$. They also do not affect dipole moment values and nonlinear optical properties. Only substitution of the amino group with the $-\mathrm{NO}_{2}$ significantly affects the batochromic shift, maximum absorption, and fluorescence, as well as nonlinear optical properties. The presence of the $-\mathrm{NO}_{2}$ group also enhances the polarity of the CT state. All derivatives exhibit a lack of bioaccumulation in the tissues of living organisms and an easy excretion with urine after fulfilling their role as a fluorescent marker. The substitution of substituents does not affect the $\mathrm{LD}_{50}$, which high value suggests no toxicity to living organisms. On the other hand, however, change of the substituent can induce hepatoxicity $\left(\mathrm{NH}_{2}\right.$ and $\left.\mathrm{NHR}\right)$ and mutagenicity $\left(\mathrm{NO}_{2}, \mathrm{OH}, \mathrm{OR}\right)$. All substituents direct the probe to the same active center (LYS114) through which the biocomplex is formed. None of the tested markers are suitable for use in two-photon studies. In addition, conjugation promotes hypsochromic shift in the position of maximum absorption and fluorescence for all analyzed markers. The smallest shift is observed for the $\mathrm{NO}_{2}$ derivative.
Also, for this substituent, conjugation slightly improves the two-photon absorption cross section value. In summary, all analyzed fluorescent probes are suitable for medical imaging applications. Nevertheless, the most valuable alternative is the $\mathrm{NO}_{2}$ derivative. Expanding its structure with additional substituents will allow obtaining valuable probes with the desired properties for use in in vivo and in vitro bioimaging.

Acknowledgments This research was supported in part by PLGrid Infrastructure.

\section{Compliance with ethical standards}

Conflict of interest The authors declare that there are no conflicts of interest.

Open Access This article is licensed under a Creative Commons Attribution 4.0 International License, which permits use, sharing, adaptation, distribution and reproduction in any medium or format, as long as you give appropriate credit to the original author(s) and the source, provide a link to the Creative Commons licence, and indicate if changes were made. The images or other third party material in this article are included in the article's Creative Commons licence, unless indicated otherwise in a credit line to the material. If material is not included in the article's Creative Commons licence and your intended use is not permitted by statutory regulation or exceeds the permitted use, you will need to obtain permission directly from the copyright holder. To view a copy of this licence, visit http://creativecommons.org/licenses/by/4.0/.

\section{References}

1. Dawood KM (2013) Benzofuran derivatives: a patent review. Expert Opin Ther Patents 23:1133-1156. https://doi.org/10.1517/ 13543776.2013.801455

2. Naik R, Harmalkar DS, Xu X, Jang K, Lee K (2015) Bioactive benzofuran derivatives: Moracins $\mathrm{A}-\mathrm{Z}$ in medicinal chemistry. Eur J Med Chem 90:379-393. https://doi.org/10.1016/j.ejmech. 2014.11.047

3. Nevagi RJ, Dighe SN (2015) Biological and medicinal significance of benzofuran. Eur J Med Chem 97:561-581. https://doi.org/10. 1016/j.ejmech.2014.10.085

4. Khanam H, Shamsuzzaman (2014) Bioactive benzofuran derivatives: a review. Eur J Med Chem 5:1-22. https://doi.org/10.1016/ j.ejmech.2014.11.039

5. Hwu JR, Chuang KS, Chuang SH, Tsay SC (2005) New benzo[b]furans as electroluminescent materials for emitting blue light. Org Lett 7:1545-1548. https://doi.org/10.1021/o1050196d

6. Xu J, Nie G, Zhang S, Han X, Pu S, Shen L, Xiao Q (2005) Electrosyntheses of poly(2,3-benzofuran) films in boron trifluoride diethyl etherate containing poly(ethylene glycol) oligomers. Euro Poly J 41:1654-1661. https://doi.org/10.1016/j.eurpolymj.2005.01. 014

7. Yang JX, Wang XL, Tu S, Xu LH (2005) Studies on the synthesis and spectral properties of novel 4-benzofuranyl-1,8-naphthalimide derivatives. Dyes Pigments 67:27-33. https://doi.org/10.1016/j. dyepig.2004.09.017

8. Anderson S, Taylor PN, Verschoor GLB (2004) Benzofuran trimers for organic electroluminescence. Chem Eur J 10:518-527. https://doi.org/10.1002/chem.200305284 
9. Tsuji H, Mitsui C, Ilies L, Sato Y, Nakamura E (2007) Synthesis and properties of 2,3,6,7-tetraarylbenzo[1,2-b:4,5-b']difurans as hole-transporting material. J Am Chem Soc 129:11902-11903. https://doi.org/10.1021/ja074365w

10. Sun YY, Liao JH, Fang JM, Chou PT, Shen CH, Hsu CW, Chen LC (2006) Fluorescent organic nanoparticles of benzofuran -naphthyridine linked molecules: formation and fluorescence enhancement in aqueous media. Org Lett 8:3713-3716. https://doi. org/10.1021/o1061293p

11. Jeon SO, Lee JY (2012) Fluorenobenzofuran as the core structure of high triplet energy host materials for green phosphorescent organic light-emitting diodes. J Mater Chem 22:10537-10541. https://doi.org/10.1039/c2jm30473b

12. Baldisserotto A, Demurtas M, Lampronti I, Moi D, Balboni G, Vertuani S, Manfredini S, Onnis V (2018) Benzofuran hydrazones as potential scaffold in the development of multifunctional drugs: synthesis and evaluation of antioxidant, photoprotective and antiproliferative activity. Eur J Med Chem 156:118-125. https://doi. org/10.1016/j.ejmech.2018.07.001

13. Hiremath SM, Suvitha A, Patil NR, Hiremath CS, Khemalapure SS, Pattanayak SK, Negalurmath VS, Obelannavar K (2018) Molecular structure, vibrational spectra, NMR, UV, NBO, NLO, HOMOLUMO and molecular docking of 2-(4, 6-dimethyl-1-benzofuran3 -yl) acetic acid (2DBAA): experimental and theoretical approach. J Mol Struct 1171:362-374. https://doi.org/10.1016/j.molstruc. 2018.05.109

14. Hiremath SM, Suvitha S, Patil NR, Hiremath CS, Khemalapure SS, Pattanayak SK, Negalurmath VS, Obelannavar K, Armakovic SJ, Armakovic S (2018) Synthesis of 5-(5-methyl-benzofuran-3ylmethyl)-3H- [1, 3, 4] oxadiazole-2-thione and investigation of its spectroscopic, reactivity, optoelectronic and drug likeness properties by combined computational and experimental approach. Spectrochim Acta A 205:95-110. https://doi.org/10.1016/j.saa. 2018.07.003

15. Hiremath SM, Patil AS, Hiremath CS, Basangouda M, Khemalapure SS, Patil NR, Radder SB, Armakovic SJ, Armakovic S (2019) Structural, spectroscopic characterization of 2-(5-methyl-1-benzofuran-3-yl) acetic acid in monomer, dimer and identification of specific reactive, drug likeness properties: experimental and computational study. J Mol Struct 1178:1-17. https:// doi.org/10.1016/j.molstruc.2018.10.007

16. Hiremathad A, Patil MR, Chethana KR, Chand K, Santos MA, Keri RS (2015) Benzofuran: an emerging scaffold for antimicrobial agents. RSC Adv 5(96):809-828. https://doi.org/10.1039/ c5ra20658h

17. Fares S, Selim KB, El-Sayed MAA, Goda FE (2017) Synthesis, biological evaluation and molecular modeling of novel benzofuran- $N$ - heterocyclic hybrids as anticancer agents. J Am Sci 13(11):65-76. https://doi.org/10.7537/marsjas 131117.07

18. Reichardt C (2004) Solvents and solvent effects in organic chemistry3rd edn. Wiley-VCH, New York

19. Basavaraj J, Sureshkumar HM, Inamdar SR, Wari MN (2016) Estimation of ground and excited state dipole moment of laser dyes C504T and C521T using solvatochromic shifts of absorption and fluorescence spectra. Spectrochim Acta A 154:177-184. https:// doi.org/10.1016/j.saa.2015.10.020

20. Desai VR, Hunagund SM, Basanagouda M, Kadadevarmath JS, Sidarai AH (2016) Solvent effects on the electronic absorption and fluorescence spectra of HNP: estimation of ground and excited state dipole moments. J Fluoresc 26:1391-1400. https://doi.org/10. 1007/s10895-016-1830-3

21. Desai VR, Sidarai AH, Hunagund SM, Basanagouda M, Melavanki RM, Fattepur RH, Kadadevarmath JS (2016) Steady state absorption and fluorescence study: estimation of ground and excited state dipole moments of newly synthesized pyridazin-3( $2 \mathrm{H}$ )-one derivatives. J Mol Liq 223:141-149. https://doi.org/10.1016/j. molliq.2016.08.015

22. Basavaraj J, Inamdar SR, Sureshkumar HM (2015) Solvents effect on the absorption and fluorescence spectra of 7-diethylamino-3thenoylcoumarin: evaluation and correlation between solvatochromism and solvent polarity parameters. Spectrochim Acta A 137:527-534. https://doi.org/10.1016/j.saa.2014.08.118

23. Raghavendra UP, Basanagouda M, Melavanki RM, Fattepur RH, Thipperudrappa J (2015) Solvatochromic studies of biologically active iodinated 4-aryloxymethyl coumarins and estimation of dipole moments. J Mol Liq 202:9-16. https://doi.org/10.1016/j. molliq.2014.12.003

24. Patil SS, Muddapur GV, Patil NR, Melavanki RM, Kusanur RA (2015) Fluorescence characteristics of aryl boronic acid derivate (PBA). Spectrochim Acta A 138:85-91. https://doi.org/10.1016/j. saa.2014.11.028

25. Muddapur GV, Patil NR, Patil SS, Melavanki RM, Kusanur RA (2014) Estimation of ground and excited state dipole moments of aryl boronic acid derivative by solvatochromic shift method. J Fluoresc 24:1651-1659. https://doi.org/10.1007/s10895-014$1452-6$

26. Kadadevarmath JS, Malimath GH, Patil NR, Geetanjali HS, Melavanki RM (2013) Solvent effect on the dipole moments and photo physical behaviour of 2,5-di-(5-tert-butyl-2-benzoxazolyl) thiophene dye. Can J Phys 91:1107-1113. https://doi.org/10. 1139/cjp-2013-0195

27. Thipperudrappa J, Biradar DS, Manohara SR, Hanagodimath SM, Inamdar SR, Manekutla RJ (2008) Solvent effects on the absorption and fluorescence spectra of some laser dyes: estimation of ground and excited-state dipole moments. Spectrochim Acta A 69:991997. https://doi.org/10.1016/j.saa.2007.06.002

28. Raikar US, Tangod VB, Mannopantar SR, Mastiholi BM (2010) Ground and excited state dipole moments of coumarin 337 laser dye. Optics Comm 283:4289-4292. https://doi.org/10.1016/j. optcom.2010.06.037

29. Maridevarmath CV, Naik L, Negalurmath VS, Basanagouda M, Malimath GH (2019) Synthesis, photophysical, DFT and solvent effect studies on biologically active benzofuran derivative: (5-methyl-benzofuran-3-yl)-acetic acid hydrazide. Chem Data Collect 21: 100221. https://doi.org/10.1016/j.cdc.2019.100221

30. Frisch MJ, Trucks GW, Schlegel GB et al (2009) Gaussian 09, Revision A.1. Gaussian, Inc., Wallingford

31. Adamo C, Scuseria GE, Barone V (1999) Accurate excitation energies from time-dependent density functional theory: assessing the PBE0 model. J Chem Phys 111:2889-2899. https://doi.org/10. $1063 / 1.479571$

32. Guido C, Caprasecca S (2016) How to perform corrected linear response calculations in G09. https://www1.dcci.unipi.it/ molecolab/tools/white-papers/pisalr/. https://doi.org/10.13140/RG. 2.1.1903.7845

33. Krawczyk P, Pietrzak M, Janek T, Jędrzejewska B, Cysewski P (2016) Spectroscopic and nonlinear optical properties of new chalcone fluorescent probes for bioimaging applications: a theoretical and experimental study. J Mol Model 22(125):1-11. https://doi. org/10.1007/s00894-016-2990-4

34. Krawczyk P, Jędrzejewska B, Pietrzak M, Janek T (2016) Synthesis, spectroscopic, physicochemical properties and binding site analysis of 4-(1H-phenanthro[9,10-d]-imidazol-2-yl)-benzaldehyde fluorescent probe for imaging in cell biology: experimental and theoretical study. J Photochem Photobiol B-Biol 164:112-122. https://doi.org/10.1016/j.jphotobiol.2016.07.044

35. Krawczyk P, Jędrzejewska B, Pietrzak M, Janek T (2017) Synthesis, photophysical properties and systematic evaluations of new phenanthroimidazole fluorescent probe for bioimaging: experimental and theoretical study. J Photochem Photobiol B-Biol 166: 74-85. https://doi.org/10.1016/j.jphotobiol.2016.11.008 
36. Krawczyk P, Jędrzejewska B, Cysewski P, Janek T (2017) Synthesis, photophysical and biological properties of a new oxazolone fluorescent probe for bioimaging: an experimental and theoretical study. Org Biomol Chem 15:8952-8966. https://doi. org/10.1039/c7ob02439h

37. Minezawa N (2014) State-specific solvation effect on the intramolecular charge transfer reaction in solution: a linear-response free energy TDDFT method. Chem Phys Lett 608:140-144. https://doi. org/10.1016/j.cplett.2014.05.104

38. Ming Tong GS, Chan KT, Chang X, Che C-M (2015) Theoretical studies on the photophysical properties of luminescent pincer gold(iii) arylacetylide complexes: the role of $\pi$-conjugation at the C-deprotonated $\left[\mathrm{C}^{\wedge} \mathrm{N}^{\wedge} \mathrm{C}\right]$ ligand. Chem Sci 6:3026-3037. https:// doi.org/10.1039/c4sc03697b

39. Slipchenko LV (2010) Solvation of the excited states of chromophores in polarizable environment: orbital relaxation versus polarization. J Phys Chem A 114:8824-8830. https://doi.org/10.1021/ jp101797a

40. Sneskov K, Schwabe T, Christiansen O, Kongsted J (2011) Scrutinizing the effects of polarization in QM/MM excited state calculations. Phys Chem Chem Phys 13:18551-18560. https:// doi.org/10.1039/c1cp22067e

41. Caricato M (2013) A comparison between state-specific and linearresponse formalisms for the calculation of vertical electronic transition energy in solution with the CCSD-PCM method. J Chem Phys 139:044116. https://doi.org/10.1063/1.4816482

42. Le Bahers T, Adamo C, Ciofini I (2011) A qualitative index of spatial extent in charge-transfer excitations. J Chem Theory Comput 7:2498-2506. https://doi.org/10.1021/ct200308m

43. Cancés MT, Mennucci B, Tomasi J (1997) A new integral equation formalism for the polarizable continuum model: theoretical background and applications to isotropic and anisotropic dielectrics. J Chem Phys 107:3032-3041. https://doi.org/10.1063/1.474659

44. Arivazhagan M, Muniappan P, Meenakshi R, Rajavel G (2013) PCM/TD-DFT analysis of 1-bromo-2,3-dichlorobenzene - a combined study of experimental (FT-IR and FT-Raman) and theoretical calculations. Spectrochim Acta A 105:497-508. https://doi.org/10. 1016/j.saa.2012.11.033

45. Boyd RW (2003) In: nonlinear optics2nd edn. Academic, London

46. Craig DP, Thirunamachandran T (1998) Molecular quantum electrodynamics: an introduction to radiation-molecule interaction Chap. 5. Dover Publications, Inc, Mineola, New York

47. Ohta K, Antonov L, Yamada S, Kamada K (2007) Theoretical study of the two-photon absorption properties of several asymmetrically substituted stilbenoid molecules. J Chem Phys 127:084504084515. https://doi.org/10.1063/1.2753490

48. Zaleśny R, Bartkowiak W, Styrcz S, Leszczynski J (2002) Solvent effects on conformationally induced enhancement of the twophoton absorption cross section of a pyridinium-N-phenolate betaine dye. A Quantum Chemical Study. J Phys Chem A 106:40324037. https://doi.org/10.1021/jp0142684
49. Olsen J, Jorgensen P (1985) Linear and nonlinear response functions for an exact state and for an MCSCF state. J Chem Phys 82: 3235. https://doi.org/10.1063/1.448223

50. Sałek P, Vahtras O, Guo JD, Luo Y, Helgaker T, Ågren H (2003) Calculations of two-photon absorption cross sections by means of density-functional theory. Chem Phys Lett 374:446-452. https:// doi.org/10.1016/s0009-2614(03)00681-x

51. DALTON A molecular electronic structure program. Release Dalton 2011 (2011), see http://daltonprogram.org/

52. LSDALTON, A linear scaling molecular electronic structure program. Release Dalton 2011 (2011), see http://daltonprogram.org

53. Potemkin V, Grishina M (2008) Principles for 3D/4D QSAR classification of drugs. Drug Discov Today 13(21-22):952-959. https://doi.org/10.1016/j.drudis.2008.07.006

54. Potemkin V, Grishina M (2008) A new paradigm for pattern recognition of drugs. J Comput Aided Mol Des 22(6-7):489-505. https://doi.org/10.1007/s10822-008-9203-x

55. Potemkin V, Pogrebnoy AA, Grishina MA (2009) Technique for energy decomposition in the study of "receptor-ligand" complexes. J Chem Inf Model 49(6):1389-1406. https://doi.org/10.1021/ ci800405n

56. Cosconati S, Forli S, Perryman AL, Harris R, Goodsell DS, Olson AJ (2010) Virtual screening with AutoDock: theory and practice. Expert Opin Drug Discovery 5:597-607

57. Forli S, Olson AJ (2012). J Med Chem 55:623-638. https://doi.org/ $10.1517 / 17460441.2010 .484460$

58. Trott O, Olson AJ (2010) AutoDock Vina: improving the speed and accuracy of docking with a new scoring function, efficient optimization and multithreading. J Comput Chem 31:455-461. https://doi. org/10.1002/jcc. 21334

59. Mueller-Dieckmann C, Panjikar CS, Tucker PA, Weiss MS (2005) On the routine use of soft X-rays in macromolecular crystallography. Part III. The optimal data-collection wavelength. Acta Crystallogr D Biol Crystallogr 61:1263-1272. https://doi.org/10. 1107/s0907444905021475

Publisher's note Springer Nature remains neutral with regard to jurisdictional claims in published maps and institutional affiliations.

Submission declaration Submitting the article "Modulation of benzofuran structure as a fluorescent probe to optimize linear and nonlinear optical properties and biological activities" to the Journal of Molecular Modeling which has not been published previously and is not under consideration for publication elsewhere, publication is approved by all authors, and it will not be published elsewhere including electronically in the same form, in English or in any other language, without the written consent of the copyright holder. 